Supplement of Saf. Nucl. Waste Disposal, 1, 153-154, 2021

https://doi.org/10.5194/sand-1-153-2021-supplement

(c) Author(s) 2021. CC BY 4.0 License.

Supplement of

\title{
Diffusive transport of uranium and americium through clay rock down to ultra-trace levels
}

\section{Daniel Glückman et al.}

Correspondence to: Daniel Glückman (daniel.glueckman@kit.edu)

The copyright of individual parts of the supplement might differ from the article licence. 
Karlsruher Institut für Technologie

\title{
Diffusion of $\mathrm{U}(\mathrm{VI})$ and Am(III) through Opalinus Clay studied down to ultra-trace levels
}

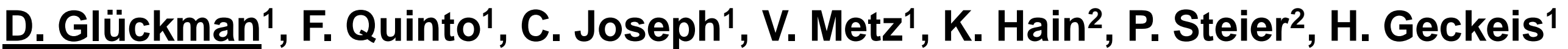

${ }^{1}$ Institute for Nuclear Waste Disposal (INE), KIT, Karlsruhe, Germany

IIsotope Physics, Faculty of Physics, University of Vienna, Vienna, Austria
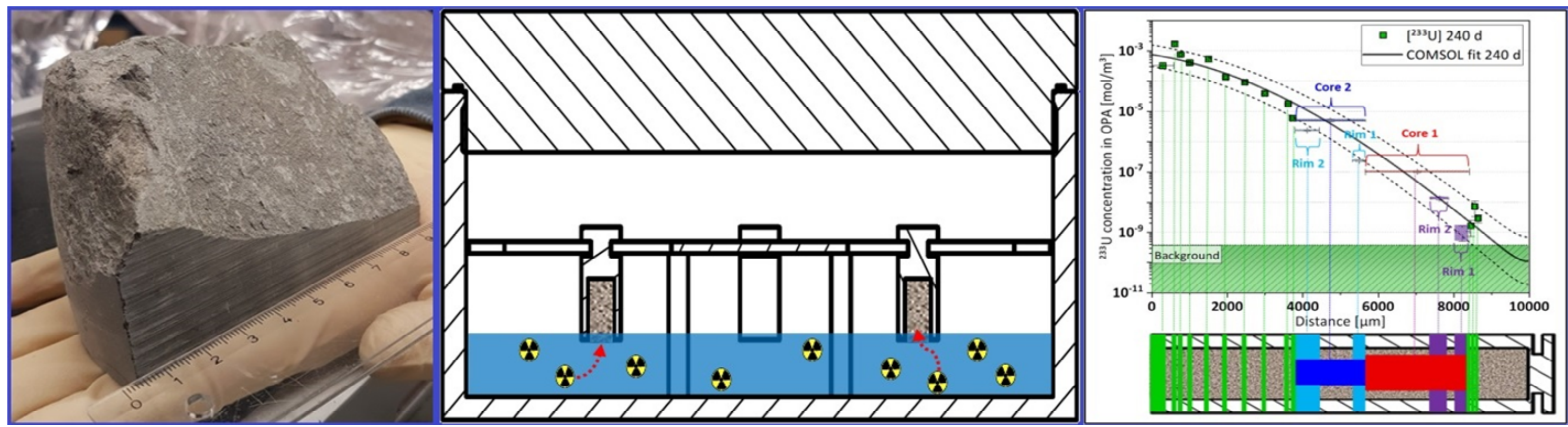


\section{Motivation}

\section{Expected scenario}

- Porewater interacts with HLW

- Radionuclides dissolve partly

- Main transport mechanism in clay rock: Diffusion

- Assumption: Actinides present mainly in low oxidation states (+III, +IV) due to reducing conditions

- Consequence: Low solubilities, strong sorption \& slow diffusion of actinides through clay rock

$>$ Ultra-trace actinide concentrations in the far-field are expected

\section{$\rightarrow$ Aim: Study of actinide diffusion in clay rock down to ultra-trace levels}

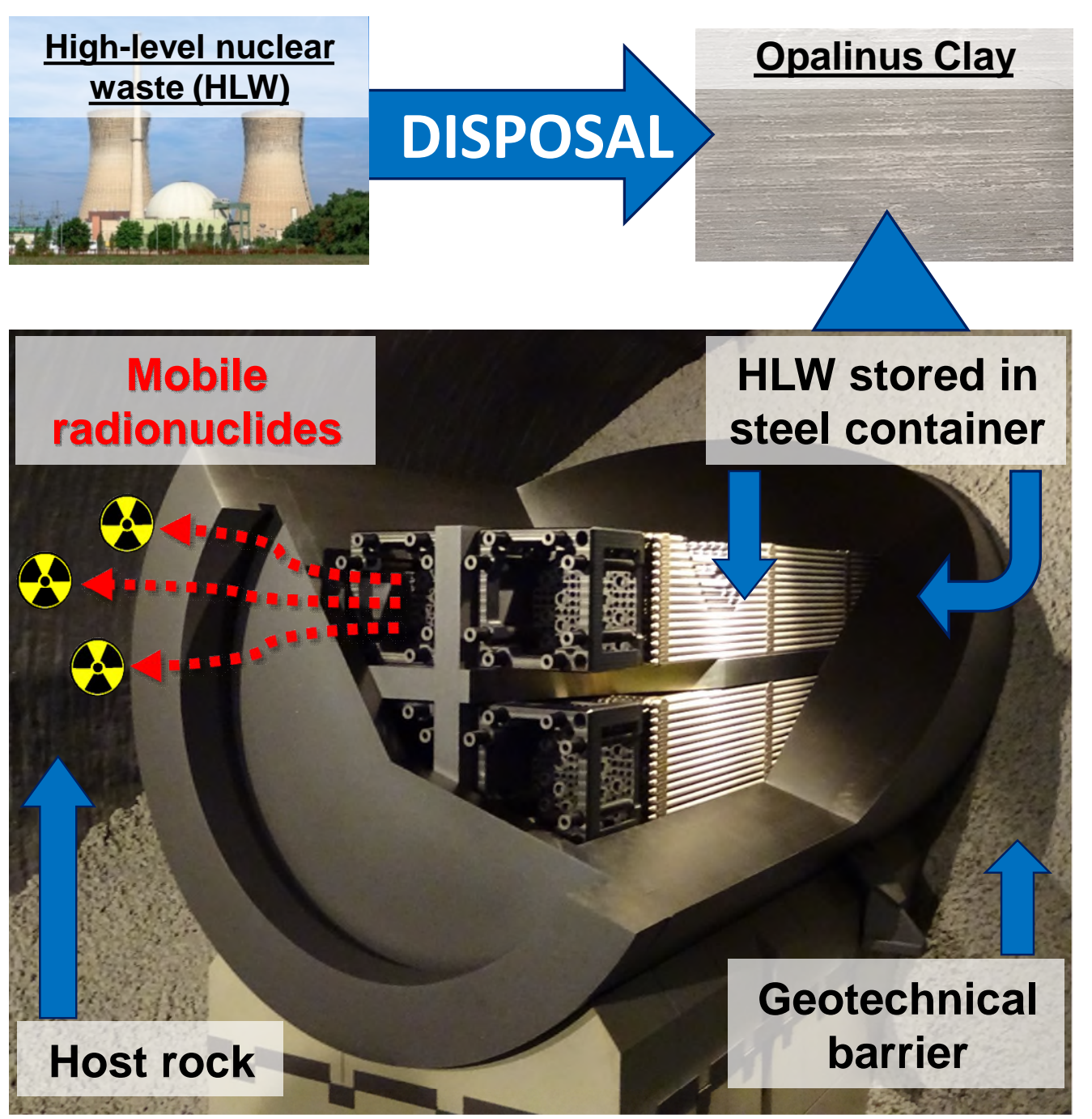




\section{Data on actinide diffusion available in literature}

\begin{tabular}{|c|c|c|c|c|}
\hline Reference & Conditions & Tracer(s) & Clay & $\begin{array}{l}\text { Limit of detection } \\
\text { (LOD) / }\left(\mathrm{mol} / \mathrm{m}^{3}\right)\end{array}$ \\
\hline Yamaguchi et al. (2007) & reducing & ${ }^{237} \mathrm{~Np}(\mathrm{IV}),{ }^{238} \mathrm{Pu}(\mathrm{IV})$ & Bentonite & $\approx 3 \times 10^{-2}$ \\
\hline Wu et al. (2009) & aerobic & ${ }^{237} \mathrm{~Np}(\mathrm{~V})$ & OPA & $\approx 7 \times 10^{-2}$ \\
\hline Joseph et al. (2013) & anaerobic & ${ }^{233} \mathrm{U}(\mathrm{VI})$ & OPA & $\approx 10^{-4}$ \\
\hline
\end{tabular}

Gap in knowledge: Diffusive behavior of actinides $\leq<10^{-4} \mathrm{~mol} / \mathrm{m}^{3} ?$

\section{Aim: Explore potential differences in diffusive behavior of} actinides at ultra-trace vs. higher concentrations 


\section{Method of choice}

- Requirement: Extremely sensitive analytical technique

- Accelerator Mass Spectrometry (AMS)

$>$ Most sensitive analytical technique for ultra-trace determination of long-lived actinides in environmental samples

\section{$\rightarrow \mathrm{LOD}_{\text {Actinides }} \approx 5 \times 10^{-19} \mathrm{~mol} / \mathrm{sample}$ in clay matrices $\approx 10^{-11} \mathrm{~mol} / \mathrm{m}^{3}\left(\approx 2 \mathrm{ng} / \mathrm{m}^{3}\right)$}
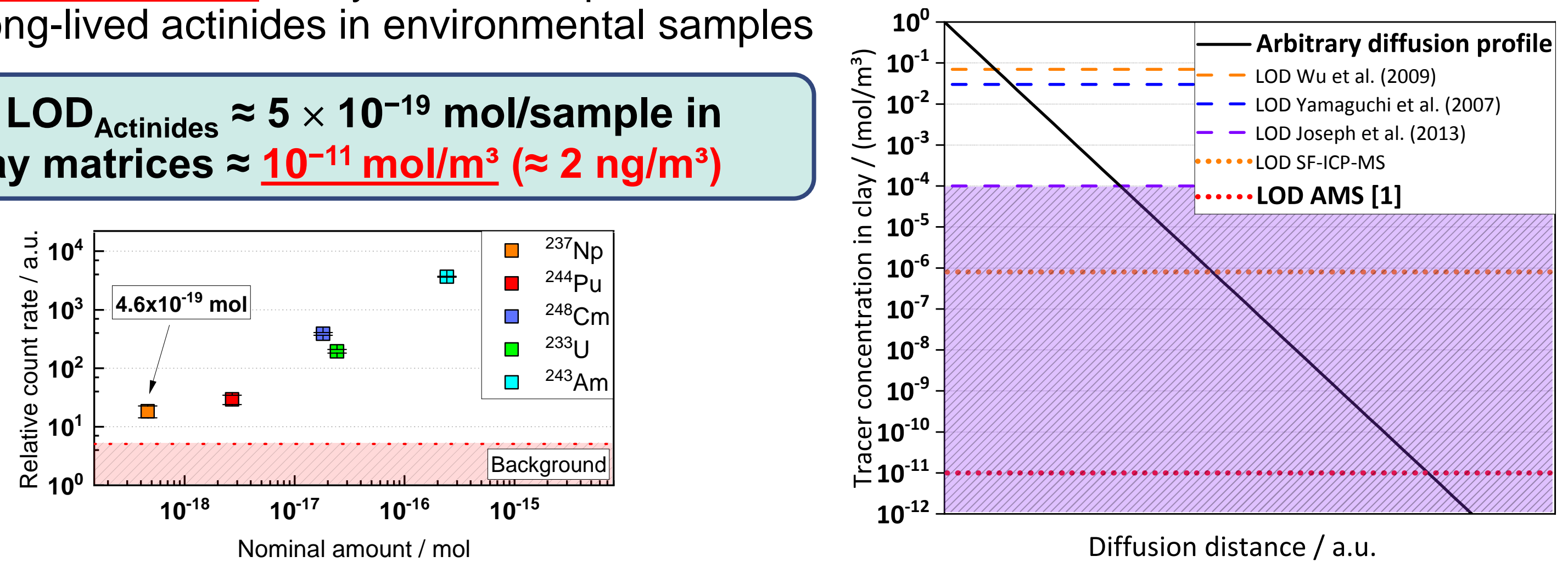

[1] Glückman, D., et al., Concurrent determination of $\mathrm{U}, \mathrm{Np}, \mathrm{Pu}, \mathrm{Am}$ and $\mathrm{Cm}$ in clay systems at ultra-trace levels with AMS (under internal revision). 


\section{In-diffusion setup}

\section{Clay rock samples}

- Opalinus Clay (OPA), shaly facies (Mont Terri, Switzerland)

- Drilled parallel to the bedding

- Preparation of cylindrical samples

- Embedded in PMMA (,Plexiglas“) sample holder with epoxy resin

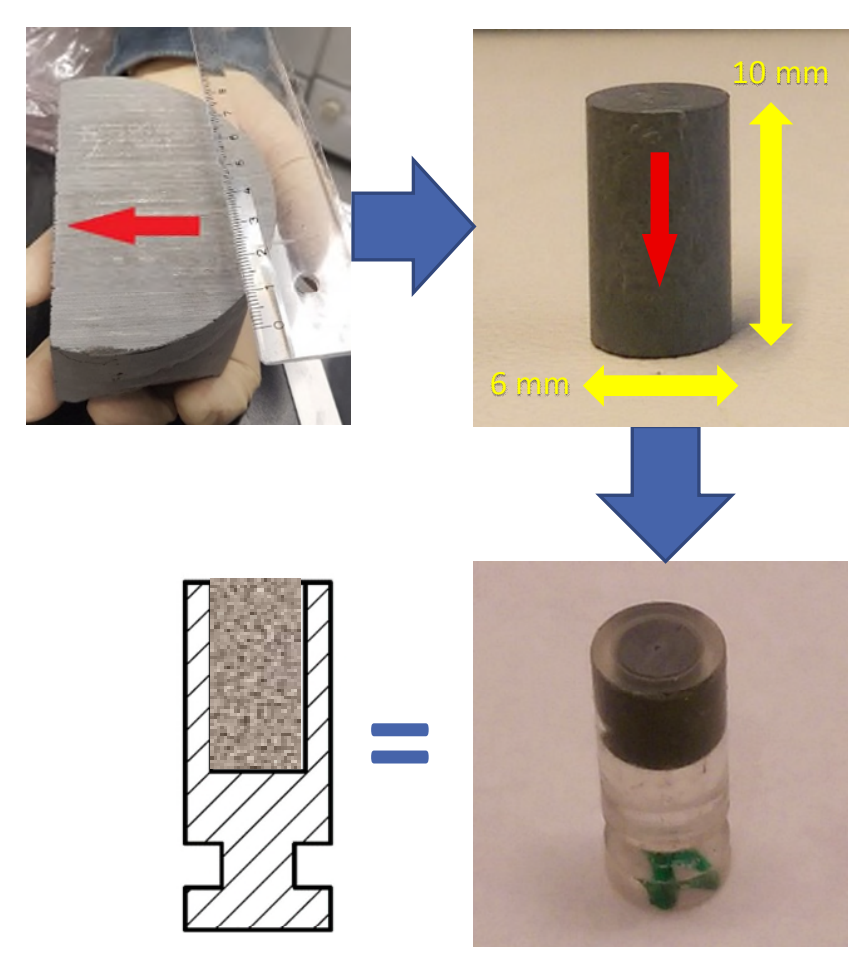




\section{In-diffusion setup}

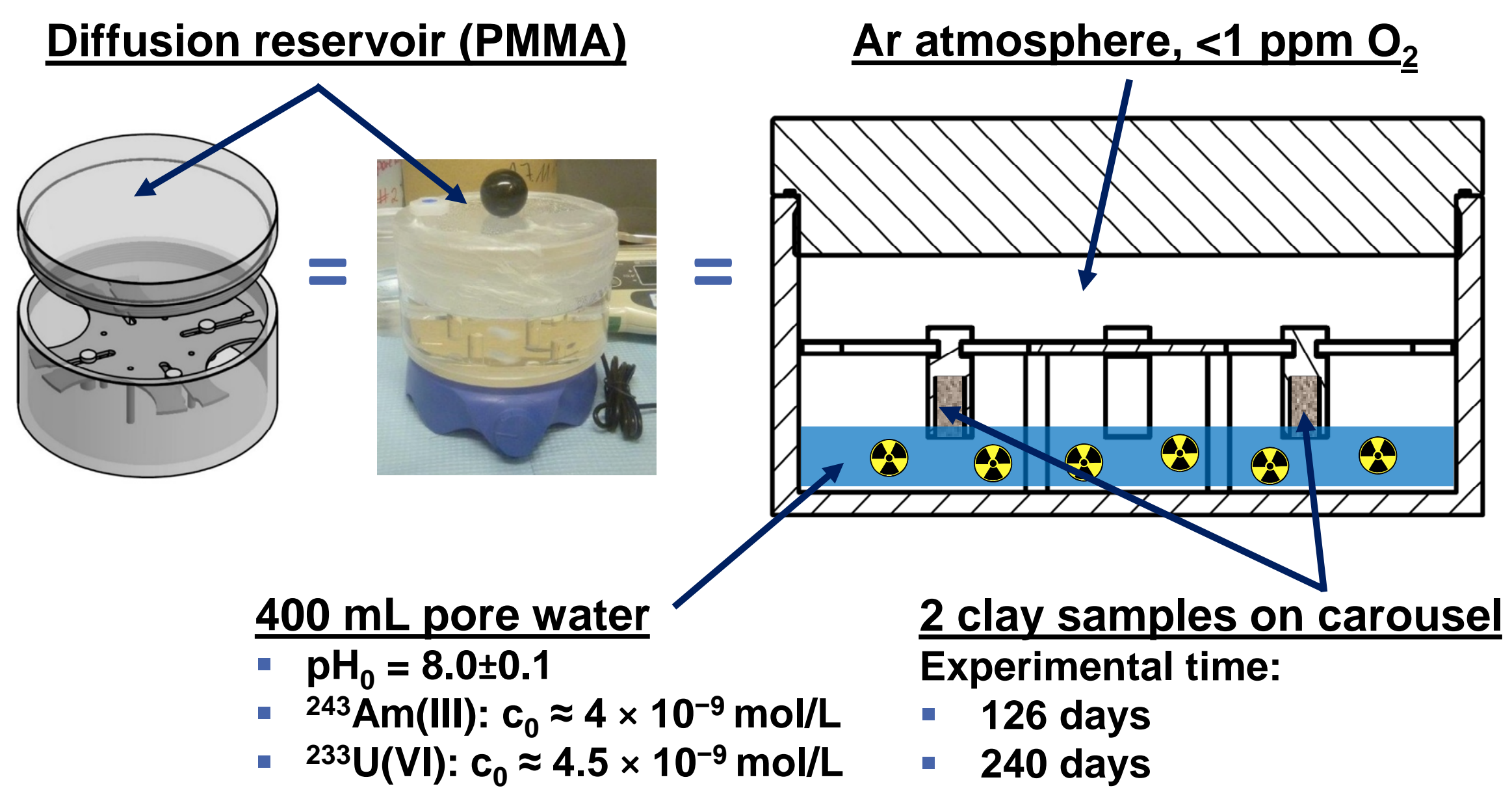

Synthetic pore water composition [1]: 


\section{Sample processing ("126 days" sample)}

- Removal of clay sample from diffusion reservoir

- PMMA cylinder glued on top (support)

- Saw off groove

- Expose back part of clay sample

$>$ Processing in direction of increasing tracer concentration by abrasive peeling [1]

„126 days“ sample:

Unexpectedly high tracer concentrations, especially for longer distances:

Cross-contamination? Preferential pathways?

$\Rightarrow$ Processing procedure for „240 days“ sample adjusted!

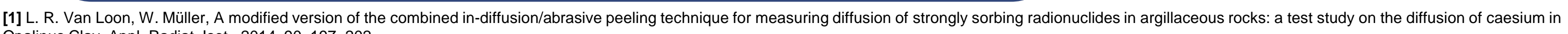
Opalinus Clay, Appl. Radiat. Isot., 2014, 90, 197-202. 


\section{Sample processing (“240 days” sample)}

- Removal of external surface $(\approx 100 \mu \mathrm{m})$

$>$ Elimination of sample holder contamination

- Drilling of core segments (red, light blue) and abrasion of corresponding rim segments (purple, dark blue)

$>$ Investigation of preferential pathways across sample rim

- Abrasion of full segments (green): Abrasive peeling
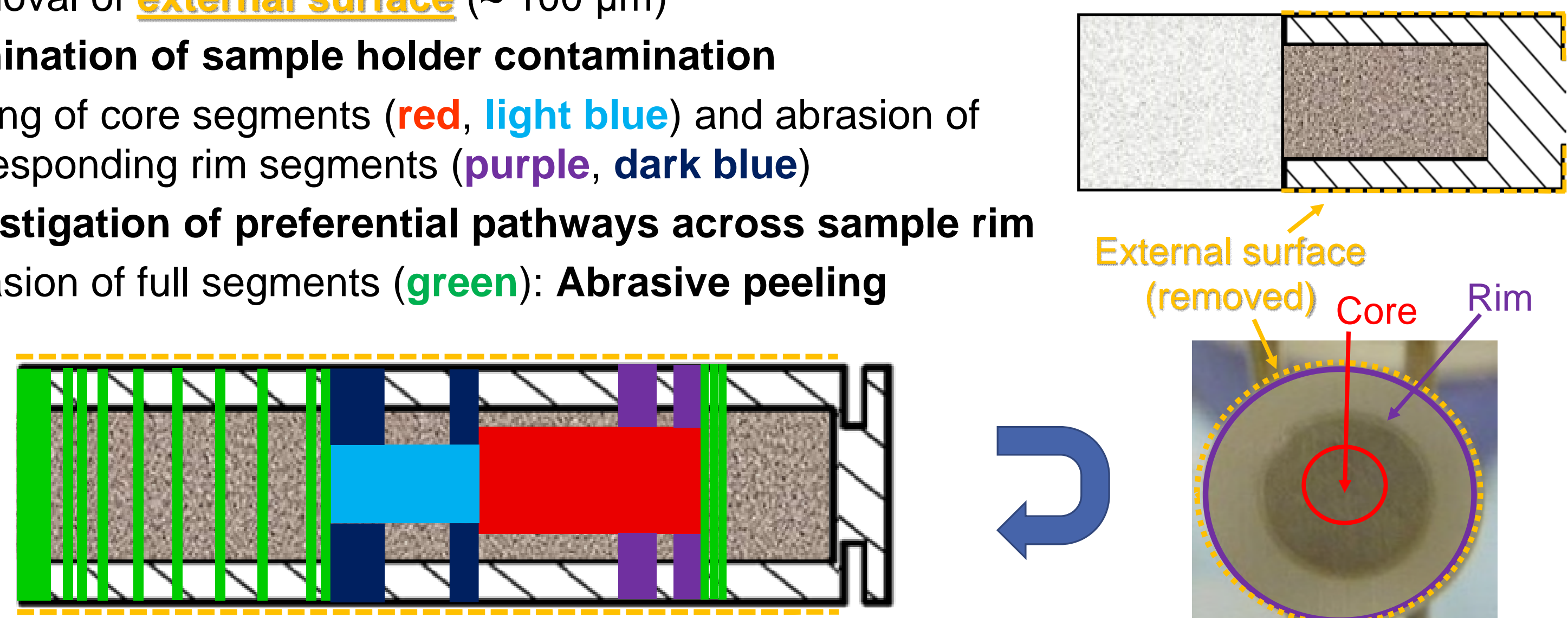

External surface

(removed)
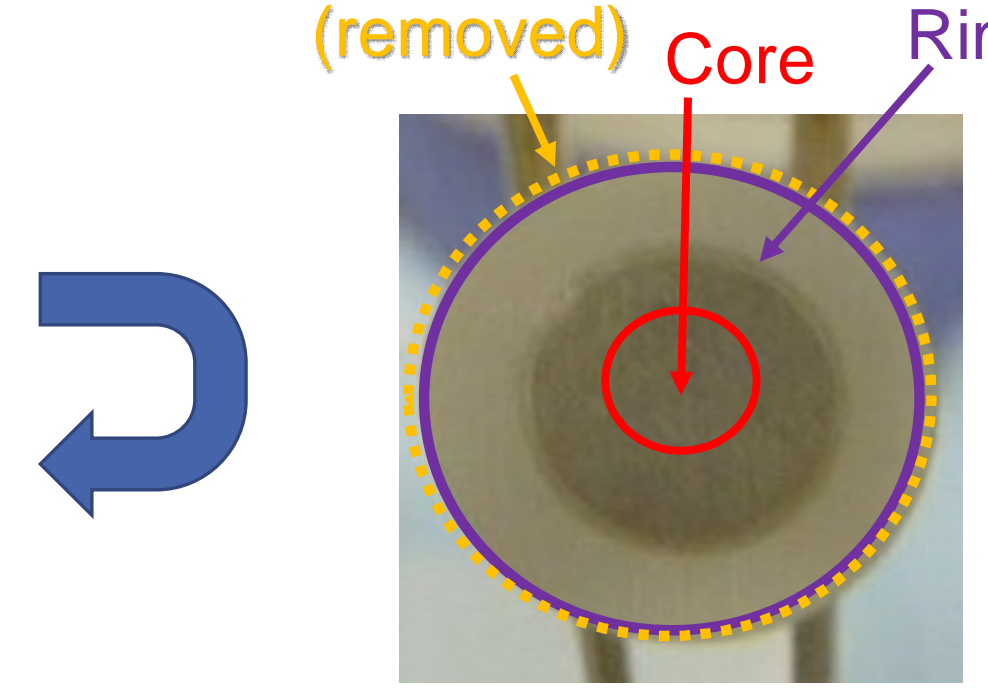


\section{Diffusion of ${ }^{233} \mathrm{U}(\mathrm{VI})$ through OPA}

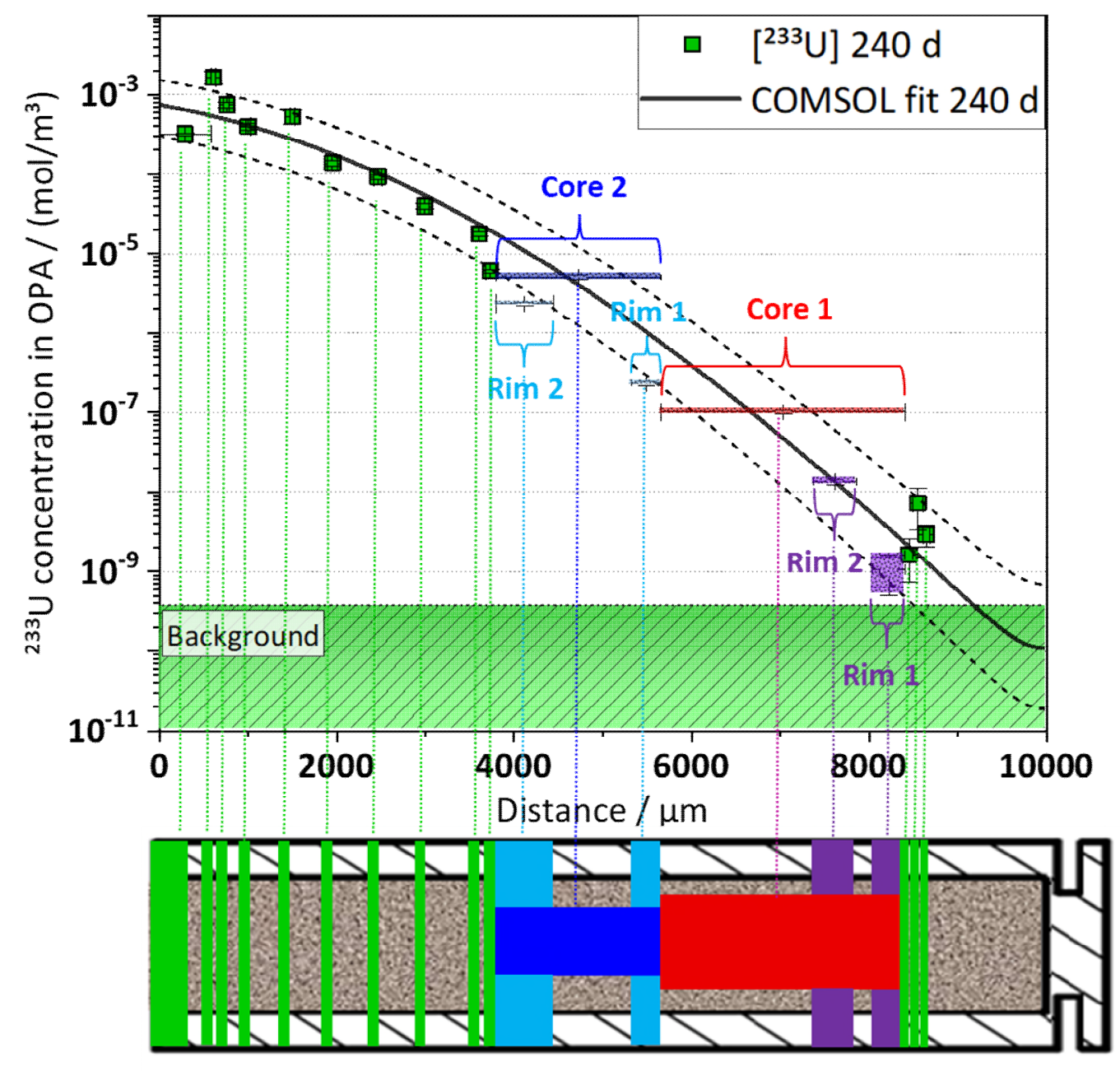

- Determination of concentration profile down to $\approx 10^{-9} \mathrm{~mol} / \mathrm{m}^{3}$

$>$ Improvement by $\underline{5}$ orders of magnitude compared to previous studies

- Core and rim segments follow general trend of decreasing ${ }^{233} \mathrm{U}$ concentration

$>$ No preferential pathway across sample rim 


\section{Diffusion of ${ }^{233} \mathrm{U}(\mathrm{VI})$ through OPA}

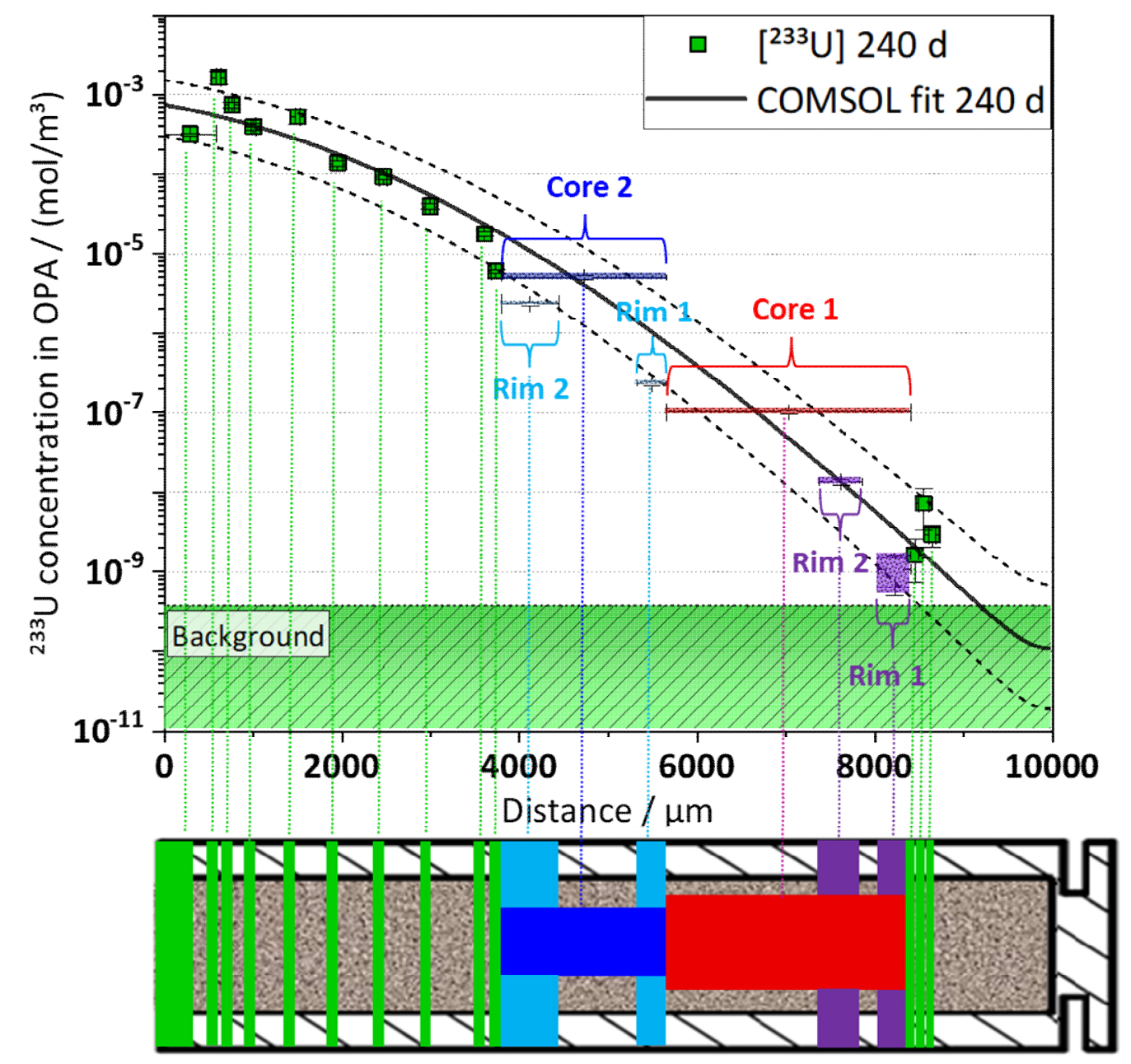

- Distribution coefficient $\boldsymbol{K}_{\mathrm{d}}$ fairly in agreement with literature data

- Effective diffusion coefficient $\boldsymbol{D}_{\mathrm{e}}$ higher due to different diffusion directions [2]

\begin{tabular}{|c|c|c|}
\hline Parameter & This work & Literature \\
\hline$K_{\mathrm{d}} /\left(\mathrm{m}^{3} / \mathrm{kg}\right)$ & $\begin{array}{c}0.105(+0.111 /- \\
0.065)\end{array}$ & $0.025 \pm 0.003[1]$ \\
\hline $\begin{array}{c}D_{\mathrm{e}} / \\
\left(\times 10^{-11} \mathrm{~m}^{2} / \mathrm{s}\right)\end{array}$ & $1.6(+2.0 /-1.1)$ & $0.19 \pm 0.04[1]$ \\
\hline Bedding & parallel & perpendicular \\
\hline $\begin{array}{l}\text { Confining } \\
\text { pressure }\end{array}$ & none & $5 \mathrm{MPa}$ \\
\hline
\end{tabular}

[1] C. Joseph et al., Diffusion of U(VI) in Opalinus Clay: Influence of temperature and humic acid, Geochim. Cosmochim. Acta, 2013, 109, 74-89.

[2] L. R. Van Loon. et al., Anisotropic Diffusion in Layered Argillaceous Rocks: A Case Study with Opalinus Clay, Environ. Sci. Technol., $2004,38,5721-5728$. 


\section{Diffusion of ${ }^{243} \mathrm{Am}(\mathrm{III})$ through OPA}

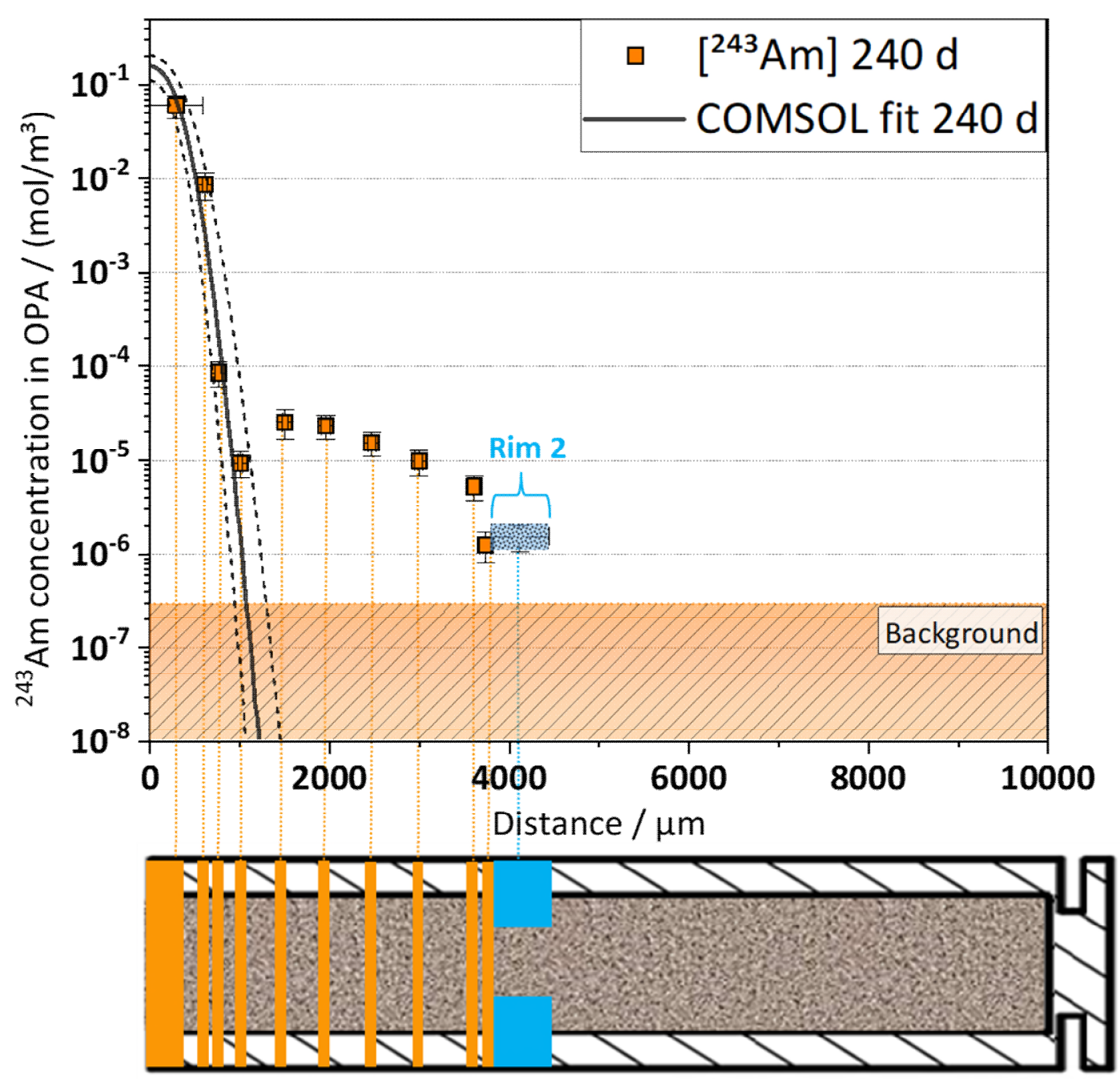

- Determination of concentration profile down to $\approx 10^{-6} \mathrm{~mol} / \mathrm{m}^{3}$

- Profile is featured by two different sections:

$>$ 0-1000 $\mu \mathrm{m}$ : Strong decrease in concentration

$>$ 1500-4000 $\mu \mathrm{m}$ : Less distinct decrease

$>$,fast runner" profile? 


\section{Diffusion of ${ }^{243} \mathrm{Am}$ (III) through OPA}

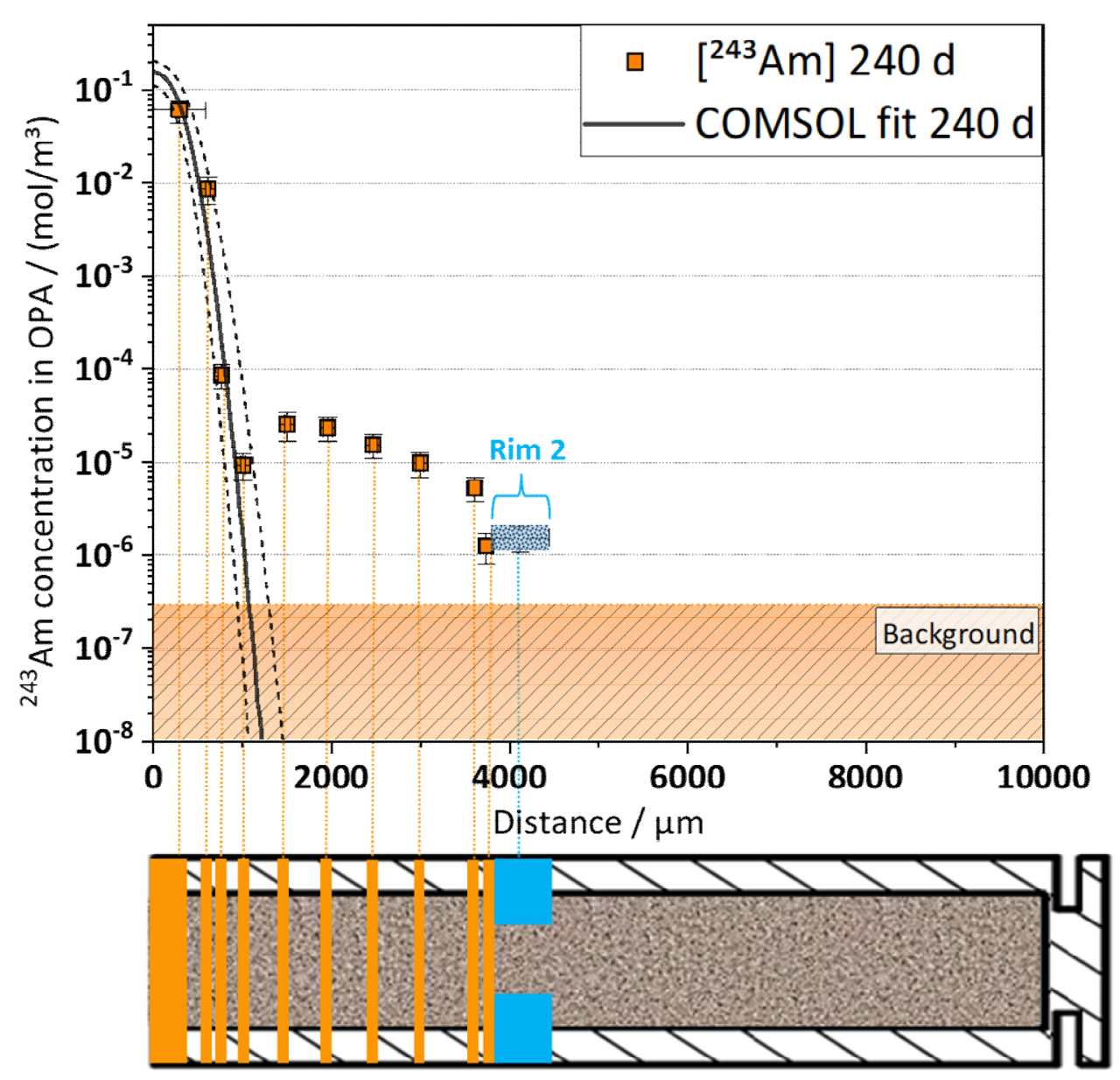

- $\boldsymbol{K}_{\mathrm{d}}$ of first profile section in fair agreement with literature data

- $D_{\mathrm{a}}$ significantly lower compared with literature data

$\Rightarrow$ Different experimental conditions

\begin{tabular}{|c|c|c|}
\hline Parameter & This work & Literature \\
\hline$K_{\mathrm{d}} /\left(\mathrm{m}^{3} / \mathrm{kg}\right)$ & $100 \pm 30$ & 63 [1] \\
\hline $\begin{array}{c}D_{\mathrm{e}} / \\
\left(\times 10^{-10} \mathrm{~m}^{2} / \mathrm{s}\right)\end{array}$ & $2.4(+2.1 /-1.1)$ & \\
\hline $\begin{array}{l}\text { Apparent diffusion coefficient } \\
D_{\mathrm{a}} /\left(\times \mathbf{1 0}^{-15} \mathrm{~m}^{2} / \mathrm{s}\right)\end{array}$ & $1.0(+0.9 /-0.5)$ & $17[2]$ \\
\hline Density / (kg/m³) & 2400 & $\frac{1600 \text { [2] }}{\text { (sand-bentonite) }}$ \\
\hline Main diffusing species & {$\left[\mathrm{Am}\left(\mathrm{CO}_{3}\right)\right]^{+}$} & {$\left[\mathrm{Am}\left(\mathrm{CO}_{3}\right)_{3}\right]^{3-}[2]$} \\
\hline
\end{tabular}

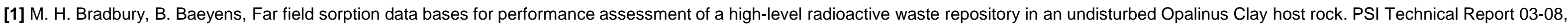
2003, Paul Scherrer Institut, Villigen, Switzerland.

[2] T. Sawaguchi, et al., Diffusion of Cs, Np, Am and Co in compacted sand-bentonite mixtures: evidence for surface diffusion of Cs cations, Clay Miner., 2013, 48, 411-422. 


\section{Comparison of $\mathrm{Am}$ (III) and Eu(III) diffusion}

\begin{tabular}{|ccc|}
\hline Parameter & This work & Literature [1] \\
\hline Tracer & $\mathrm{Am}(\mathrm{III})$ & $\mathrm{Eu}(\mathrm{III})$ \\
\hline $\begin{array}{c}\text { Initial tracer } \\
\text { concentration / (mol/L) }\end{array}$ & $4 \times 10^{-9}$ & $1 \times 10^{-8}$ \\
\hline Diffusion time / d & & \\
\hline $\boldsymbol{K}_{\mathrm{d}} /\left(\mathbf{m}^{\mathbf{3}} / \mathbf{k g}\right)$ & 240 & 200 \\
\hline $\boldsymbol{D}_{\mathrm{a}} /\left(\mathbf{\times} \mathbf{1 0}^{-15} \mathbf{~ m}^{\mathbf{2}} / \mathbf{s}\right)$ & $100 \pm 30$ & 60 \\
\hline
\end{tabular}
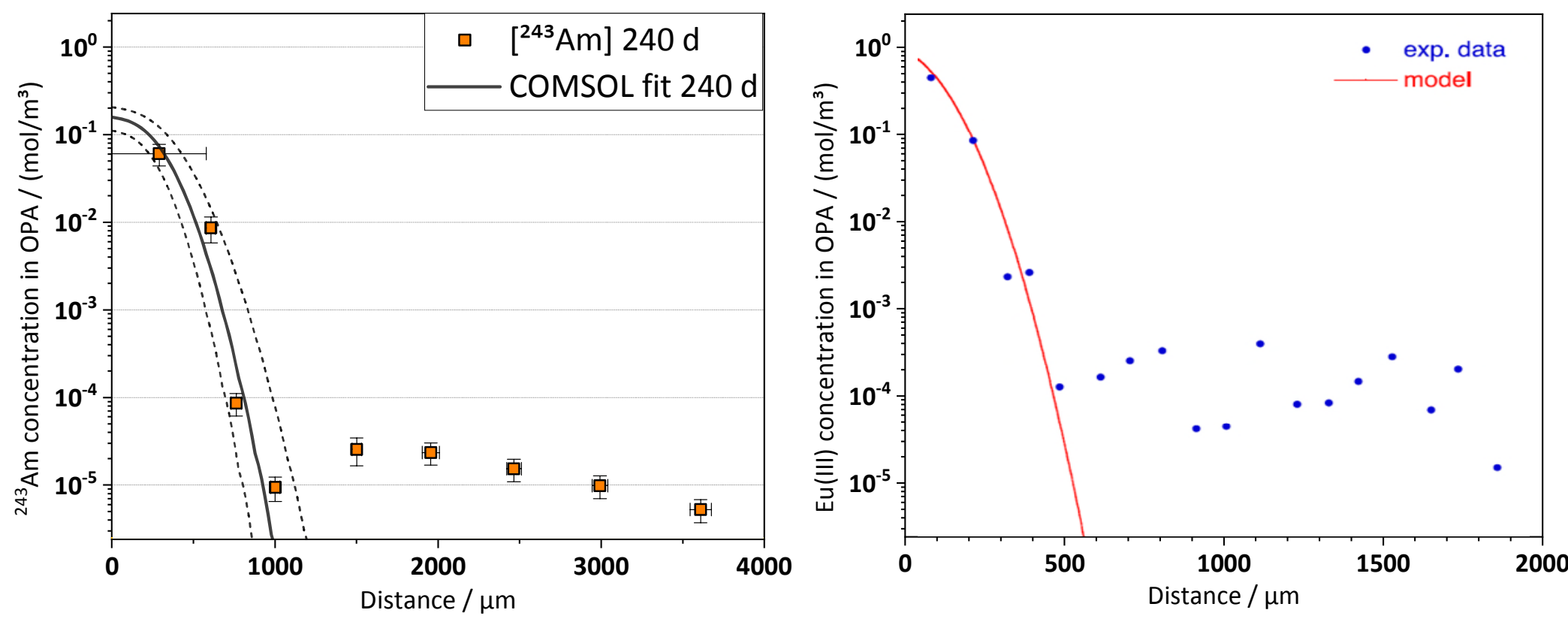


\section{$\mu \mathrm{CT}$ investigation of clay rock sample}

KIT IAM-ET: Adrian Lindner, Dr. Wolfgang Menesklou, Dr. Jochen Joos

\section{Is the „fast runner" profile caused by micro cracks?}

- Abundance of cracks increases in direction of the proximal sample side

- Crack diameter up to $\mathbf{5 0} \boldsymbol{\mu m}$

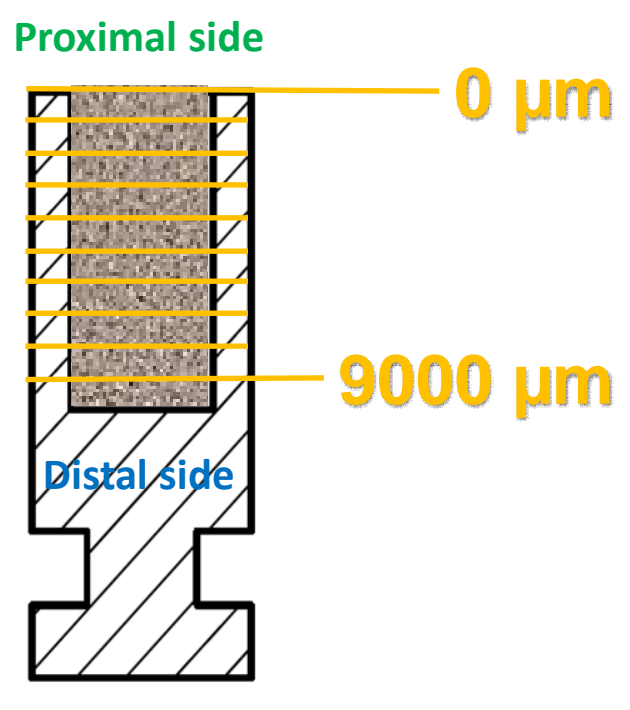

\section{Hypothesis:}

Tracers were transported by a combination of:

- Pore diffusion $\rightarrow$ through porous clay matrix

- Water diffusion $\rightarrow$ along cracks (faster process!) 


\section{Outlook}

- Ongoing short-term diffusion experiments with ${ }^{233} \mathrm{U}(\mathrm{VI})$ and ${ }^{243} \mathrm{Am}$ (III) for $20,40 \& 60 \mathrm{~d}$

- Setup of COMSOL Multiphysics model including crack geometry

\section{$\Rightarrow$ Goal: Investigation of „fast-runner“ profiles}




\section{Acknowledgements}

- KIT INE: Workshop

- University Mainz: Prof. Tobias Reich

- PSI: Martin Glaus, Luc Van Loon

- KIT IAM-ET: Adrian Lindner, Wolfgang Menesklou, Jochen JoOS

- RADIATE project $\rightarrow$ Funding of AMS beamtime

- Federal Ministry of Education and Research \& Helmholtz Association of German Research $\rightarrow$ Funding of the iCROSS project (contract No.: 02 NUK $053 \mathrm{C}$ )

\section{Thank you for your attention!}

